\title{
IDENTIFICATION AND MEASUREMENT OF PASTES RHEOLOGICAL PROPERTIES - EFFECTS OF WATER DISSOCIATION
}

\author{
A. Poitou, G. Racineux and N. Burlion \\ Laboratoire de Mécanique et Technologie, ENS de Cachan, CNRS, UPMC, \\ 61 avenue du président Wilson, 94235 Cachan Cedex, France
}

\begin{abstract}
Sewage sludges exhibit in most cases theological properties which do not allow us to classify them either as simple fluids or as solids. On one hand, they are not simple fluids because of their non-Newtonian, shearthinning and time-dependent (thixotropic or rheopectic) behaviour and because of the possibility for some of them of showing a yield stress. On the other hand, they can hardly be considered as solids, mostly because of their high sensitivity to velocity and because they are practically very often pumped as if they were fluids. This intermediate state sometimes referred to as paste state is in fact very common and is extensively studied, from a manufacturing point of view in areas like ceramics, food or polymer processing and from a civil engineering point of view in soil mechanics for the determination of clay theology. Many experimental tests have already been designed for their characterisation. The aim of this presentation is to compare some of them and try to make a synthesis between the engineering rheology and soil mechanics approaches.

For this purpose we show experimental results on the same material with different rheometric devices: viz (i) a tube (or capillary) ROSAND rheometer, (ii) a triaxial soil mechanics cell, (iii) an oscillatory, stress driven, plane and plane STRESSTECH rheometer, and (iv) a specific squeezing device which has been designed here following an idea introduced by Laun for polymers. The triaxial cell and the plane and plane theometers are similar in the sense that they are supposed to allow for an homogeneous state of stress and strain (or rate of strain). In contrast, the tube or squeezing test does not allow for a homogeneous mechanical state. This means that the first two tests enable a real measure of the constitutive relation but in limited conditions while the two others provide a way to identify rheological parameters in flow conditions which are close to the process conditions. The main originalities of this presentation are (i) the balanced point of view between the fluid and the solid mechanics approach and (ii) the method by which the contact properties of the paste is measured with the lube and the squeezing rheometer, giving evidence of a water migration inside the paste.
\end{abstract}

\section{KEYWORDS}

Capillary; filtration; pastes; rheometry; sludges; squeezing test; triaxial cell.

\section{INTRODUCTION}

The experimental devices devoted to the measurement of rheological properties of pastes can be roughly divided into 3 main categories. In the first category, we find the identification tests for which the mechanical state is homogeneous. These tests do not give a deep understanding of the constitutive behaviour of the material, but are often very helpful as normalised procedures to screen, in a rather easy way, some global characteristics of a paste. Hundreds of them have been designed for many years but among them, the 
penetrometer, the texturometer or the scissometer are, for good reasons, well adapted to characterising the cohesiveness of sludges in a paste state. In a second group of devices, we find some tests designed to allow for a homogeneous state of stress and rate of strain inside the sample. These machines are often more expensive and more limited, but allow for a real measurement of the constitutive behaviour whereas this behaviour can at best only be identified with machines of the first group. In a third group at last, we find experiments which exhibit heterogeneous mechanical states but which allow, with some assumptions that are usually not too restrictive, a direct measurement of one stress and one (rate of) strain at a given point inside the sample. The capillary test associated with Rabinowitch corrections as well as a squeezing test analysed with a new procedure are of this kind.

The aim of this paper is to discuss experimental results that were obtained on a colloidal paste made of titanium oxide in an aqueous binder. These results are intended to give a good idea of what can be obtained on materials like limed sludges. For this purpose, we carried out mechanical test of the two last groups in order (i) to discuss the expression of the real three-dimensional constitutive behaviour of the paste and (ii) to evidence the main characteristic features of this type of material. The most important property is certainly their capability to exhibit a water dissociation induced by the flow. The main originality of this paper lies in the wide variety of experiments that were carried out (from polymer rheology to soil mechanics) as well as in a description of typical difficulties which are encountered in testing these materials. We do not give a real mathematical modelling of this behaviour which is in our opinion still an open problem.

\section{TEST PROVIDING A POSSIBLE HOMOGENEOUS STATE OF STRESS}

Pastes behave qualitatively like a viscoplastic material which is often characterised at low shear rates with two classical tests which are more or less used respectively in soil mechanics applications and in polymer engineering studies.

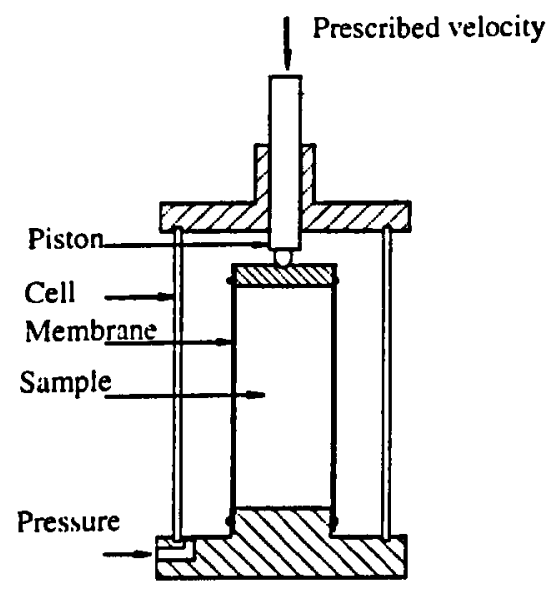

Figure 1. Triaxial cell.

\section{Soilmechanics triaxial cell}

In this experiment (Figure 1), a cylindrical sample is subjected to a radial pressure $p$ and then charged axially with a displacement of the upper plate at a constant velocity. The resultant vertical force acting on the upper plate is measured, which enable the determination of the stress $q$ in either drained or undrained conditions. The behaviour of soil-like materials is sketched in Figure 2 and that of a non-linear viscous fluid is shown in Figure 3. For plastic materials the maximum $q$ of the curve measured for different values of the pressure $p$ allows drawing of the plastic yield surface $(p, q)$ of the material (Coulomb, Cam Clay, ...). For nearly incompressible materials (which is the case of wet sludges), this curve turns out to be nearly 
independent of $\mathrm{p}$, leading to a Mises- or Tresca-like behaviour. For viscous materials in contrast, the curves $q$ versus the axial deformation depend strongly on the velocity. The determination of these curves for different velocities provides a way to measure for example the Norton behaviour.

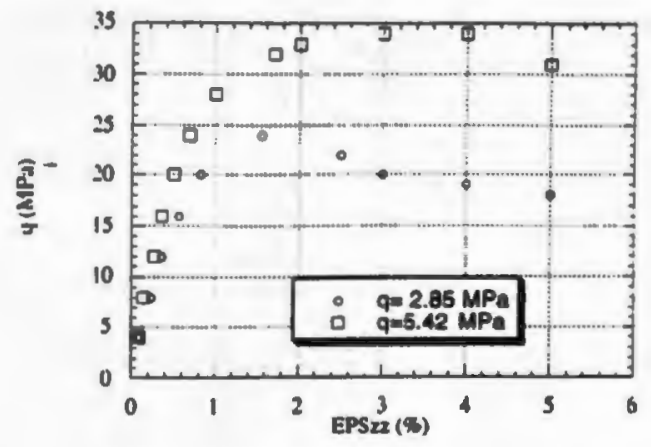

Figure 2. Typieal plot for soils or dy sludges

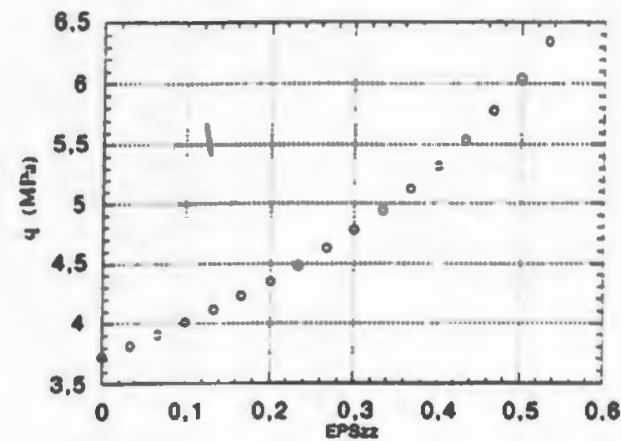

Figure 3. Behaviour of a non-linear viscous fluid.

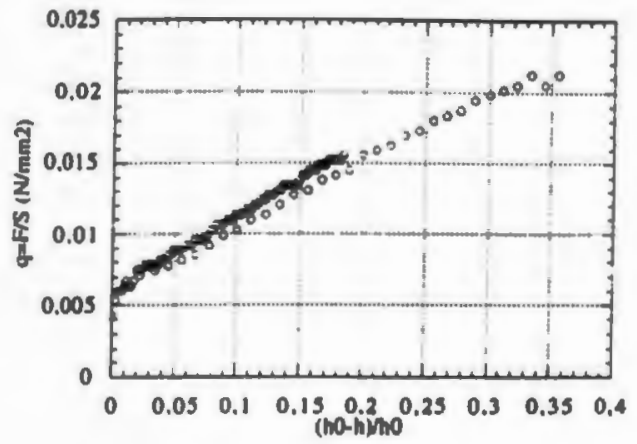

Figure 4. Results for a paste.

Typical results obtained on pastes are shown in Figure 4. From these results, it can be deduced that (i) their is no maximum in the curve $q$ versus axial deformation indicating a different behaviour if compared to a soil, (ii) the behaviour is not very time-dependent. 


\section{The plane-plane rheometer}

The plane-plane experiments are difficult to carry out on pastes. If sliding problems can be avoided, in making use of rough surfaces for the plates, some localisation of strain can be evidenced as sketched in Figure 5. This behaviour could be characteristic of a plastic material but the threshold which is measured with a stress driven rheometer is far from reproducible. This makes the experiments hardly tractable from a practical point of view.

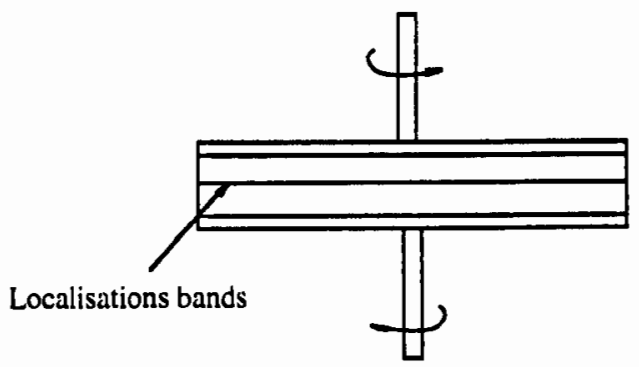

Figure 5. Typical localisation bands for the plane-plane experiment.

\section{Discussion}

These experiments are expensive compared with other normalised tests. Their only interest lies in their capability to provide a homogeneous situation allowing for a direct measurement of the paste behaviour. However, because of the loss of density homogeneity in the triaxial cell, and because of the loss of strain homogeneity in the plane-plane experiment, their utilisation is less attractive for pastes than for other more standard materials.

\section{SQUEEZING TEST}

In a squeezing test, the state of stress is both heterogeneous and time-dependent, if the experiment is carried out, as it is classical, at a constant velocity (for example, Leider and Bind, 1974; Gartling and Phan Thien, 1984; Lanos 1993). However in recent paper (Laun 1996; Laun et al., 1997) dealing with polymeric materials, it has been shown that this test can be driven in a more clever way. If the velocity $h$ is driven in order to keep the quantity $h_{-} F$ constant during the test, it can be shown that under assumptions similar to those of Rabinovitch corrections (possibly non-linear viscous, incompressible, homogeneous material with an eventual viscous sliding), that the shear stress at the surface at the edge of the disc is constant during the test. This is similar to the fact that in a capillary rheometer, the wall stress is constant. With this procedure, it is thus possible to use a penetrometer-like device with the same measurement goals as in a triaxial cell for example (but for a much wider range of strain rates).

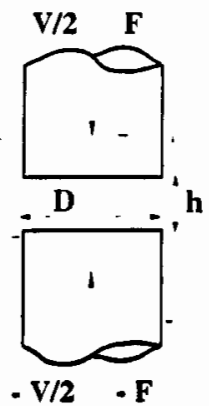

Figure 6. Squeezing test device. 


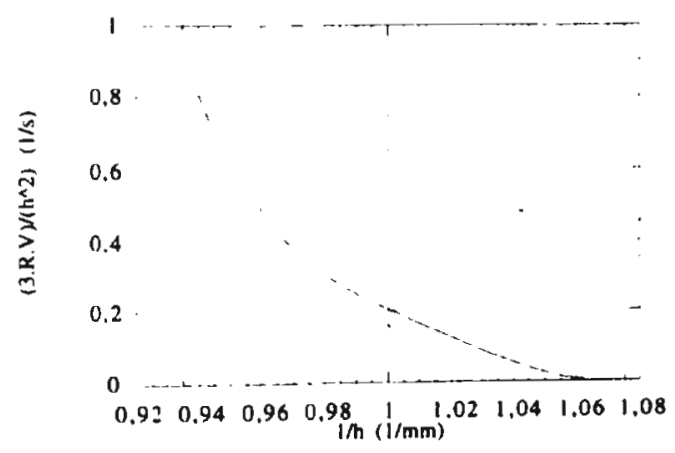

Figure 7. Experimental result for h $F=400 \mathrm{~N} \mathrm{~mm}$.

This test was carried out on our material on a servo-hydraulic MTS tensile machine equipped with parallel circular plates of diameter $D=20 \mathrm{~mm}$. The automatic control is achieved with the MTS-TEST STAR software. The sample is formed by a first compression at a constant velocity $\mathbf{h}$; so that its initial thickness is $h_{0}$ (figure 6). Figure 7 shows a typical plot obtained for a prescribed value h $F=400 \mathrm{~N} \mathrm{~mm}$. This result evidences clearly a deviation form a standard viscoplastic behaviour. The main reason for this deviation can be found qualitatively in noting that at the end of the test, the specimen is clearly drier at the centre than at the edge, which explains that as the material does not remain homogeneous, the Laun analysis is no longer valid. A confirmation of this analysis can be found in performing the test at a constant velocity. If the material was homogeneous and of Norton's type, the squeezing force would be:

$$
F=2 \pi\left(\frac{2 m+1}{m}\right)^{m} \frac{1}{(m+3)} \frac{K}{\sqrt{2}(m \cdot 1)} \frac{V^{m}}{h^{2 m+1}} R^{(m+3)}
$$

Equation 1 would indicate that when $m$ is positive, $F$ increases with $\mathbf{h}$, even if, for $m<1$ (shear-thinning materials), the mechanical work needed for this deformation decreases with h. Figure 8 shows results obtained for a given initial thickness $h_{0} . F$ is measured as a function of $h$ at different velocities. These results evidence an opposite behaviour which confirms the migration of water inside the sample.

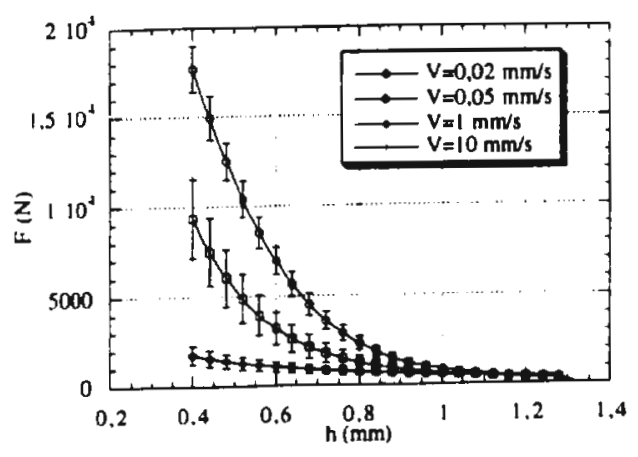

Figure 8. Influence of for a given initial thickness $h_{0}$

\section{CAPILLARY RHEOMETRY}

The tests have been carried out on a capillary ROSAND RH 7 - 2 rheometer. In order to prevent oxidation and friction of the grains of the different surfaces, the barrel is made of stainless steel and the piston of 
PTFE. Water loss cannot be avoided with a classical polymer melts rheometer. In order to prevent them at the pressure transducer as well as at the capillary fixing device we used o-ring joints. This procedure is needed to get reproducible results. The experiments are carried out at a constant prescribed velocity so that the apparent shear rate ranges from $10 \mathrm{~s}^{-1}$ to $20000 \mathrm{~s}^{-1}$. For each prescribed flow rate $Q$, one measures the pressure drop $(\Delta \mathrm{p})$ mes between the entry and the exit after stabilisation of the pressure (Figure 9 ).

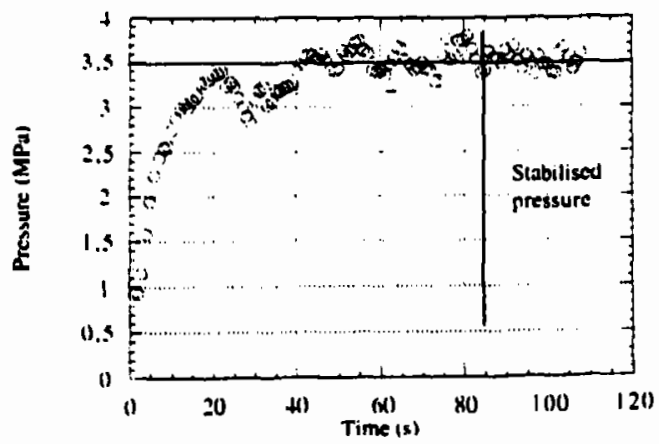

Figure 9. Pressure measured for a given flow rate before stabilisation.

Previous studies on similar materials have evidenced wall effects (Geiger, 1989; Wiegreffe, 1991; Lanteri el al., 1996; Talbi, 1993; Mourniac et al., 1992). In order to study these effects we carried out different tests with various surface/volume ratios of the specimen. In our case this means that it was interesting to use capillaries of different diameters. After proper entry corrections, the apparent flow curves can be plotted as shown in Figure 10. For a classical homogeneous viscous incompressible and sticking material, these curves should be the same. The absence of master curve is typical of pastes and has been described in previous papers (Lanteri et al., 1996) as proof of the dissociation of the material. It can be shown that a sort of parietal water layer appear whose thickness depends on the radius of the capillary. This layer is responsible for what could called an apparent siding effect.

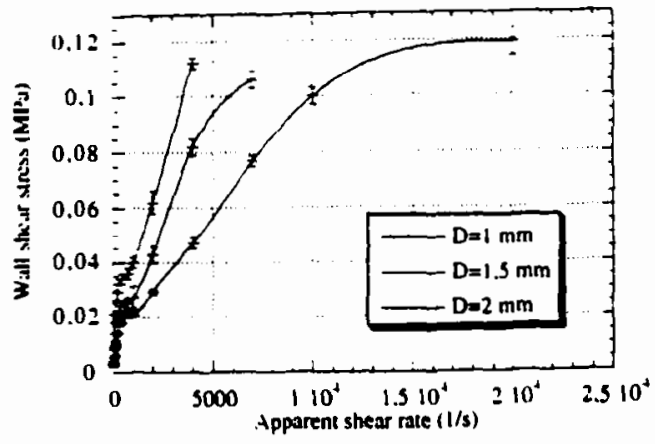

Figure 10. Apparent flow curve for different capillary diameters

This dissociation is confirmed here by plotting the entry pressure measured with the Bagley correction at different velocities. For each diameter it is thus possible to determine the pressure drop inside the capillary as well as entry pressure inside the die. By this way we evidenced results which are qualitatively similar to those previously described in figure 8 for the squeezing test: (i) at low flow rates, the pressure drop inside the capillary is of the same magnitude as the entry pressure (Figure 11), (ii) at higher flow rates, the entry pressure decreases with the velocity (Figure 12), which confirms that the water migrates if the characteristic time of the lest is less than the characteristic time needed for the water to migrate. 


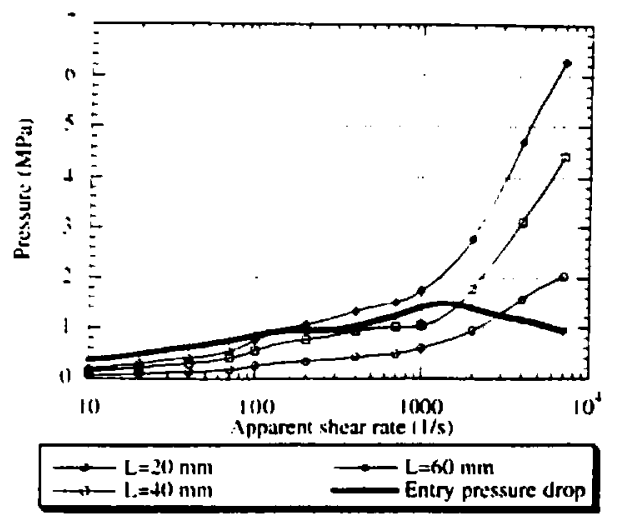

Figure I1. Pressure drop inside capillaries and measured entry pressure, $D=1.5 \mathrm{~mm}$.

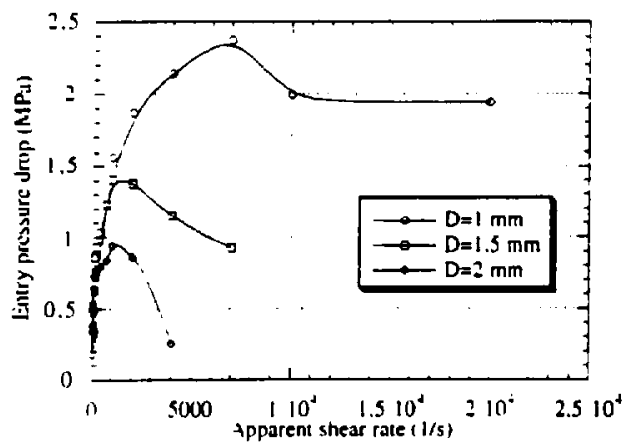

Figure 12. Entry pressure (converging zone of the die) - influence of velocity and diameter.

\section{CONCLUSION}

The rheological tests presented in this paper establish some results that characterise pastes' behaviour.

The tests usually chosen because they provide a homogeneous state of stress are not really adapted to pastes because they lead in fact for pastes to a loss of homogeneity (in composition or because of strain localisation for example). This makes a difference between sludges and soils as soon as we are interested in flow properties (high shear rates and steady flow conditions for example) rather than in static consolidation-like conditions.

The flow of pastes induces an heterogeneity (water migration). Even a slight variation in cessity can have strong consequences for their behaviour. We showed in this paper that this migration is not only induced by sedimentation.

Though we didn't carry out any normalised tests like penetrometry or scissometry, these tests are clearly indicated to qualify the behaviour of the pastes. But because of the lack of any real justified threedimensional mathematical modelling of the materials' behaviour, particular attention must be paid if one wants to extrapolate these normalised results to make predictions under other loading conditions. For example a direct determination of a viscosity from a scissometer measurement is not really valid if, as we have shown here, the material exhibits an induced heterogeneity capability. 
Particular care must be taken with capillary measurements. It is absolutely necessary in measuring different capillary diameters. Even if this is not usual for polymers, it is here necessary because it allows to check whether the sticking conditions are valid or whether any partetal layer is induced by the flow.

The squeezing test as well as the capillary test are certainly indicated in making a measurement of the water filtration at high shear rates under flow conditions.

\section{BIBLIOGRAPHY}

Bagley, E. B. (1957). End Corrections in the Capıllary Flow of Polyethylene Journal of Applied Physics, 28(5), $624-627$.

Gartling, D. and Phan Thien, N. (1984). A numerical simulation of plastic fluids in parallel plate plastometer. Journal of nonNewtonian fluid mechanics, 14, 347-360.

Geiger, K. (1989). Rheologische charakterisierung von EPDM Kautschukmischungen mittels kapillars-rheometers systemen. Kautschuk + Gummi kunstoffe, pp. 273-283.

Lanos, C. (1993). Methode d'identification non viscométrique de comportements de fluides, PhD thesis INSA de Rennes, France.

Lantéri, B., Burlet, H., Poitou, A. and Campion, I. (1996). Rheological behaviour of a polymer-ceramic blend used for injection moulding. J. Materials Science, 31, 1751-1760.

Laun, H. M., Neumayer, A. and Hassager, O. (1997). Determination of partial wall slip of polymer melts with squeezing flow. To be published in Rheologica acta.

Laun, H. M. (1996). Squeezing flow theometry to determine viscosity, wall slip and yield stresses of polymer melts, 12th annual meeting of the Polymer Processing Society, Sorrento.

Leider, J. and Bird, B. (1974). Squeezing flow between parallel disks I. Theoretical analysis. Ind. Eng. Chem. Fund, 13.

Leider, J. (1974). Squeezing flow between parallel disks II. Experimental results. Ind. Eng. Chem. Fund, 13.

Mooney, M. and Black, S. A. (1952). A generalized Fluidity Power-Law and Laws of Extrusion. Journal of Colloid Science, 7 , 204-217.

Mourniac, Ph., Agassant, J. F. and Vetgne, B. (1992). Determination of the wall slip velocity in the flow of a SBR compound. Rheologica Acta, 31, 565-574.

Talbi, I. (1993). Comportement rhéologique de suspensions colloïdales concentrées monodispersées, PhD thesis, Pierre et Marie Curie university, France.

Wiegreffe. S. (1991). Untersuchungen zum Wandgleitverhalten von EPDM und SBR. Kautschuk + Gummi Kunstoffe, 44, 216221. 